\title{
Therapeutic Hypothermia for Perinatal Asphyxia in Low-Resource Settings
}

\author{
B. Vishnu Bhat ${ }^{1}$ D $\cdot$ B. Adhisivam ${ }^{2}$
}

Received: 9 August 2021 / Accepted: 9 August 2021 /Published online: 1 September 2021

(c) Dr. K C Chaudhuri Foundation 2021

Hypoxic-ischemic encephalopathy (HIE) continues to be a major cause of neonatal mortality and morbidity globally, especially in low- and middle-income countries (LMIC). Therapeutic hypothermia has been proven to be effective in reducing morbidity associated with HIE and has become the standard of care for HIE in developed countries. However, in low-resource settings, where the problem is more common, therapeutic cooling is still in the nascent phase [1].

Krishnan et al. have recently published a review article titled "Rise and Fall of Therapeutic Hypothermia in LowResource Settings: Lessons from the HELIX Trial" in the Indian Journal of Pediatrics [2]. The title itself is misleading as there has been an increasing awareness regarding therapeutic cooling over the years and many centers in India have been practicing the technique with good results and there has never been a fall in the practice of cooling. However, this particular review article written in the context of LMIC appears entirely biased and filled with despicable allegations. It portrays scientific research in LMIC in poor light using inappropriate language. The HELIX (hypothermia for encephalopathy in low- and middle-income countries) study did not observe any difference in the primary outcome [3]. However, the study had 17 predefined secondary outcomes and the authors have presented only unadjusted analysis raising serious concerns about the statistical handling of multiple comparisons and presenting an exaggerated view based on inappropriate analyses $[3,4]$.

According to a recent systematic review, therapeutic hypothermia reduces the risk of death in neonates with moderate-to-severe hypoxic-ischemic encephalopathy.

B. Vishnu Bhat

drvishnubhat@yahoo.com

1 Director-Medical Research, Aarupadaiveedu Medical College and Hospital, Vinayaka Mission's Research Foundation-DU, Puducherry 609602, India

2 Department of Neonatology, Jawaharlal Institute of Postgraduate Medical Education and Research (JIPMER), Puducherry, India
This benefit is also seen in low- and middle-income countries. Based on the evidence, therapeutic hypothermia should be offered as part of routine clinical care to newborns with hypoxic-ischemic encephalopathy especially in low- and middle-income countries [5]. A Cochrane review by Jacobs et al. also noted that therapeutic hypothermia reduces mortality without increasing major disability in survivors. The benefits of cooling on survival and neurodevelopment outweigh the short-term adverse effects. Hypothermia should be instituted in term and late preterm infants with moderate-to-severe HIE if identified before $6 \mathrm{~h}$ of age [6].

The authors have self-proclaimed that HELIX is the only phase III clinical trial in LMIC belittling all the research work done in LMIC including that in CMC, Vellore and Jawaharlal Institute of Postgraduate Medical Education and Research (JIPMER), Puducherry in this area [7-16]. It is mentioned in the review that between 2012 and 2020, six randomized controlled trials (RCTs) were reported from JIPMER, Puducherry. The authors have also alleged "Despite similar inclusion criteria, mortality varied between 7 and $50 \%$ in the usual care group in these six trials, raising concerns about the reliability of these data." The study by Joy et al. with lowest mortality (7\%) among control had (12\%) infants with severe encephalopathy [8]. However, the study by Tanigasalam et al. with highest mortality among control had $25 \%$ of infants with severe encephalopathy and hence the higher mortality [10]. Without analyzing the minor details, the authors have questioned the reliability of data.

The authors claim, without substantiating, that phase changing material (MiraCradle) used in India is wrongly marketed with commercial interest as lifesaving and innovative device. The innovation has been included in WHO compendium of innovative health technologies [17]. The Indian scientists involved in this innovation declared in the publication, which is in public domain, that they provided concept and design without any competing interest for development of MiraCradle [13]. 
The authors have mentioned, "In LMIC, clinicians neither have dedicated time to conduct high-quality research, nor have access to experienced clinical trials units. Hence, most LMIC trials are conducted by postgraduate students with little prior research experience, and often as a part of their dissertation while undertaking full-time clinical work. Poor understanding of the research methodologies, inadequate research supervision, use of databases without audit trails, single person data management, time pressures from clinical work, proliferation of predatory journals, and ambition to get quick publications with positive results-all contribute to research fraud and generate data that may cause harm." To make such sweeping statement on the research quality in LMIC shows biased interpretation of research and researchers who are trying to answer problems faced in LMIC and provide innovations that can improve child survival in resource-limited settings.

It is strange that the Indian centers with adequate experience in cooling were not included in the HELIX trial. The study sites chosen may have access to 3 Tesla MRI scanners and spectroscopy but that does not imply that their care for the study participants would match the standard of care and experience available in centers which are routinely cooling babies. Contrary to the results of a systematic review $[5,6]$ and studies reported from various sites in India [7-16], higher mortality and morbidity in the HELIX trial need to be explored. In the HELIX trial, chronic hypoxic changes in MRI were recorded. These could be related to the delayed recruitment or poor condition of the infants on arrival. Therapeutic hypothermia can cause harm in such infants with pre-existing clinical instability. It is unclear whether any interim analysis was performed in the HELIX trial. The harmful effect observed could have been picked up early, and ethically, the trial should have been stopped by the Data and Safety Monitoring Board. The authors of the review (2) have clearly gone overboard in congratulating themselves on this trial and using inappropriate language to criticize previous work in the field without any substantive evidence, violating the basic principles of scientific writing.

Authors of the HELIX trial conclude that therapeutic cooling should not be continued in LMIC. This statement and the trial itself needs a close examination before it can be taken as the final word for a modality that is being used in high-income countries. Therefore, we recommend that therapeutic hypothermia can be continued in centers with the requisite facilities and manpower. Data including long-term neurodevelopment from all centers doing cooling routinely should be collated and published.

\section{Declarations}

Conflict of Interest None.

\section{References}

1. Bhat BV, Adhisivam B. Therapeutic cooling for perinatal asphyxia-Indian experience. Indian J Pediatr. 2014;81:585-91.

2. Krishnan V, Kumar V, Shankaran S, et al. Rise and fall of therapeutic hypothermia in low-resource settings: lessons from the HELIX trial. Indian J Pediatr. 2021. https://doi.org/ 10.1007/s12098-021-03861-y.

3. Thayyil S, Pant S, Montaldo P, et al. Hypothermia for moderate or severe neonatal encephalopathy in low-income and middleincome countries (HELIX): a randomised controlled trial in India, Sri Lanka, and Bangladesh. Lancet Glob Health. 2021. https://doi.org/10.1016/S2214-109X(21)00264-3.

4. Aneja S, Sharma S. Hypoxic ischaemic encephalopathy in low resource settings-time to stop cooling? Lancet Glob Health. 2021. https://doi.org/10.1016/S2214-109X(21)00343-0.

5. Abate BB, Bimerew M, Gebremichael B, et al. Effects of therapeutic hypothermia on death among asphyxiated neonates with hypoxic-ischemic encephalopathy: a systematic review and meta-analysis of randomized control trials. PLoS One. 2021;16:e0247229.

6. Jacobs SE, Berg M, Hunt R, Tarnow-Mordi WO, Inder TE, Davis PG. Cooling fornewborns with hypoxic ischaemic encephalopathy. Cochrane Database Syst Rev. 2013;2013:CD003311.

7. Bharadwaj SK, Bhat BV. Therapeutic hypothermia using gel packs for term neonates with hypoxic ischaemic encephalopathy in resource-limited settings: a randomized controlled trial. J Trop Pediatr. 2012;58:382-8.

8. Joy R, Pournami F, Bethou A, Bhat VB, Bobby Z. Effect of therapeutic hypothermia on oxidative stress and outcome in term neonates with perinatal asphyxia: a randomized controlled trial. J Trop Pediatr. 2013;59:17-22.

9. Gane BD, Bhat V, Rao R, Nandhakumar S, Harichandrakumar $\mathrm{KT}$, Adhisivam B. Effect of therapeutic hypothermia on DNA damage and neurodevelopmental outcome among term neonates with perinatal asphyxia: a randomized controlled trial. J Trop Pediatr. 2014;60:134-40.

10. Tanigasalam V, Bhat V, Adhisivam B, Sridhar MG. Does therapeutic hypothermia reduce acute kidney injury among term neonates with perinatal asphyxia?-a randomized controlled trial. J Matern Fetal Neonatal Med. 2016;29:2545-8.

11. Rakesh K, Bhat VB, Adhisivam B, Ajith P. Effect of therapeutic hypothermia on myocardial dysfunction in term neonates with perinatal asphyxia - a randomized controlled trial. J Matern Fetal Neonatal Med. 2018;31:2418-23.

12. Catherine RC, Bhat VB, Adhisivam B, Bharadwaj SK, Palanivel C. Effect of therapeutic hypothermia on the outcome in term neonates with hypoxic ischemic encephalopathy-a randomized controlled trial. J Trop Pediatr. 2021;67:fmaa073.

13. Thomas N, Abiramalatha T, Bhat VB, et al. Phase changing material for therapeutic hypothermia in neonates with hypoxic ischemic encephalopathy - a multi-centric study. Indian Pediatr. 2018;55:201-5.

14. Aker K, Støen R, Eikenes L, et al. Therapeutic hypothermia for neonatal hypoxic-ischaemic encephalopathy in India (THIN study): a randomised controlled trial. Arch Dis Child Fetal Neonatal Ed. 2020;105:405-11. 
15. Shabeer MP, Abiramalatha T, Smith A, et al. Comparison of two low-cost methods of cooling neonates with hypoxic ischemic encephalopathy. J Trop Pediatr. 2017;63:174-81.

16. Thomas N, George KC, Sridhar S, Kumar M, Kuruvilla KA, Jana AK. Whole body cooling in newborn infants with perinatal asphyxial encephalopathy in a low resource setting: a feasibility trial. Indian Pediatr. 2011;48:445-51.
17. World Health Organization. WHO compendium of innovative health technologies for low-resource settings. Available at: https:// apps.who.int/iris/rest/bitstreams/1153419/retrieve. Accessed on 7 Aug 2021.

Publisher's Note Springer Nature remains neutral with regard to jurisdictional claims in published maps and institutional affiliations. 